\title{
DETECTION OF THE SPATIAL VARIATIONS OF LOCAL POPULATIONS FROM THE VIEWPOINT OF URBAN STRUCTURE ANALYSIS
}

\author{
K. Kumagai ${ }^{1,}{ }^{*}$, Y. Kameda ${ }^{2}$ \\ 1 Setsunan University, 17-8, Ikeda-Nakamachi, Neyagawa, Osaka 572-8508 Japan - kumagai@civ.setsunan.ac.jp \\ 2 Setsunan University, 17-8, Ikeda-Nakamachi, Neyagawa, Osaka 572-8508 Japan - 151015ky@gmail.com
}

KEY WORDS: Local population dynamics, Population Decline, Densely Populated Area, Urban spongification, Spatial Autocorrelation.

\begin{abstract}
:
In Japan, population decline is one of the important issues that need to be tackled in socioeconomic fields. We apply an analysis method composed of a spatial autocorrelation analysis to the local population data generated through the 1995 and 2015 national census, and try to detect their spatial dynamics in this study. Through making experimental verification of the distance parameter of the spatial autocorrelation analysis, we newly define 2 indices with respect to the size and area where lower local populations are distributed in urbanized areas. It is shown that the local population dynamics are described by the difference of the 2 indices among 1995 and 2015. The expansion of areas where local population decline occurred seems to be detected by our proposed approach.
\end{abstract}

\section{BACKGROUND}

In Japan, population decline is one of the important issues that need to be tackled in socioeconomic fields (National Institute of Population and Social Security Research, 2012, Kutsuzawa, 2016). Population decline in a rapidly aging society results in the creation of many problems such as depopulation in rural areas, expansion of lower population density in urban areas, as well as a boost in spending for the maintenance of public transportation and infrastructure under the pressure of lower revenue. From the viewpoint of urban structure, the Japanese government takes several measures against it based on the concept called "Compact Plus Network". One of the measures implemented is a land use strategy for living space and urban function. In this measure, Residence Attraction Districts and Urban Function Attraction Districts are defined as core areas for maintaining sufficient population density given current and predicted population dynamics (Ministry of Land, Infrastructure, Transport and Tourism, 2014). However, it is necessary to cope with "Urban spongification" which is the current and future problem with respect to the spatial heterogeneity of local populations around the districts. "Urban spongification" is a phenomenon in which vacant land and vacant houses in urbanized areas randomly occur. This phenomenon is considered as a cause for concern about an impediment to maintaining sufficient population density because of the difficulty of integrated urban redevelopment due to the randomness. High levels of vacancies reinforcing the run-down of the districts could potentially occur under the phenomenon (Ministry of Land, Infrastructure, Transport and Tourism, 2019). It is therefore desirable to perform monitoring for the population distribution spatially. The spatial scale and variation of the local population distributions, however, have not been analysed. In this study, we apply an analysis method composed of a spatial autocorrelation analysis to the local population data generated through the 1995 and 2015 national census, and try to detect their spatial features of population dynamics.

${ }^{*}$ Corresponding author

\section{DATA AND METHODOROGY}

\subsection{Study Area}

The areas of Osaka and Kagawa prefecture were adopted as the study areas. Figure 1 shows the location of these study areas. Osaka prefecture is located in the west part of Japan. There is a second largest city in the Osaka prefecture, while a lot of satellite cities are located around the city. Local populations are distributed in various spatial patterns in the study area because there are completely urbanized areas, urbanized areas, and suburban areas. Kagawa prefecture is located in Shikoku which is an island of southern Japan. There are some core urbanized areas and large suburban areas in the Kagawa prefecture. Both prefectures have wide flat fields, and their size of land are much the same (around $1,900 \mathrm{~km}^{2}$ ).

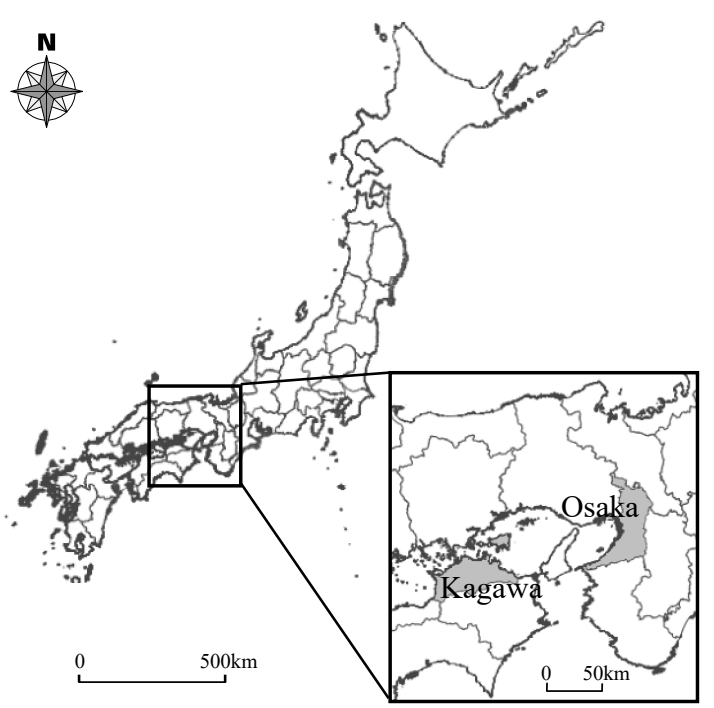

Figure 1. Study areas and their locations. The grey areas in the enlarged view of the map show Osaka and Kagawa prefecture. 


\subsection{Population Data}

The basic unit block population data of the Population Census of Japan in 1995 and 2015 were applied to the spatial analysis of demographic dynamics. The Population Census data is one of the results of the National Census. The basic unit block population data has the highest spatial resolution based on the management of National Census investigators. The total population of Osaka prefecture was 8,797,268 in 1995 and $8,839,469$ in 2015 , while that of Kagawa prefecture was $1,027,006$ in 1995 and 976,263 in 2015. We generated 60m-by$60 \mathrm{~m}$ mesh data as local population data by counting the basic unit block population data on a mesh-by-mesh basis.

\subsection{Methodology}

The spatial analysis method of local population distributions we have developed consists of a spatial autocorrelation analysis and an overlay analysis. The $G$ statistics we applied as the spatial autocorrelation analysis is described as

$$
G_{i}(d)=\frac{\sum_{i} w_{i j}(d) h_{j}}{\sum_{i} h_{i}}
$$

where is $G_{i}$ is $\mathrm{G}$ statistics, $h_{j}$ is the local population located in the area, $w_{i j}$ is a symmetric binary spatial weight matrix with ones for all links defined as being within distance $d$ of a given $i$; all other links are coded zero, including the link of a point $i$ to itself (Getis 1992). If the null hypothesis is that the set of $h_{j}$ values within $d$ of location $i$ is a random sample, we derive the $Z$ value described in Eq. (2).

$$
Z_{i}(d)=\frac{G_{i}(d)-E\left[G_{i}(d)\right]}{\sqrt{\operatorname{VarG}_{i}(d)}}
$$

Positive or negative spatial autocorrelation is obtained depending on whether the $Z$ value is positively or negatively greater than specific level of significance (Ord and Getis, 1995). As the result of the statistical tests with a significance level of $10 \%$, the area of interest was divided into the three kinds of results of the statistical test: a positive spatial autocorrelation, no spatial autocorrelation, and a negative spatial autocorrelation. Positive spatial autocorrelation means that a clustering of larger local populations occurs within distance $d$ of a given $i$.

\subsection{Application of Spatial Autocorrelation Analysis}

Kumagai et al. $(2011,2017)$ examined the fluctuation of the ratio of correlation areas to no-correlation areas with increasing distance $d$ using NDVI derived from Landsat OLI data. Figure 2 shows the fluctuation of spatial correlation/no spatial correlation areas depending on the distance parameter $d$ for local populations in Kagawa 2015 as an instance. The red and blue areas in Figure 2 mean positive and negative spatial autocorrelation areas, respectively, while the grey areas are no spatial correlation areas. As $d$ is increasing, it is indicated that positive and negative spatial correlation areas are increasing. The figure shows that both of correlation areas spread to the nocorrelation areas with increasing $d$.

For analysing the range of distance $d$, the differences of area percentages between contiguous distances were calculated as $\Delta a$. Especially, relationship between the positive spatial autocorrelation areas and the distance needs to be clarified for the spatial analysis of "Urban spongification". Figure 3 shows

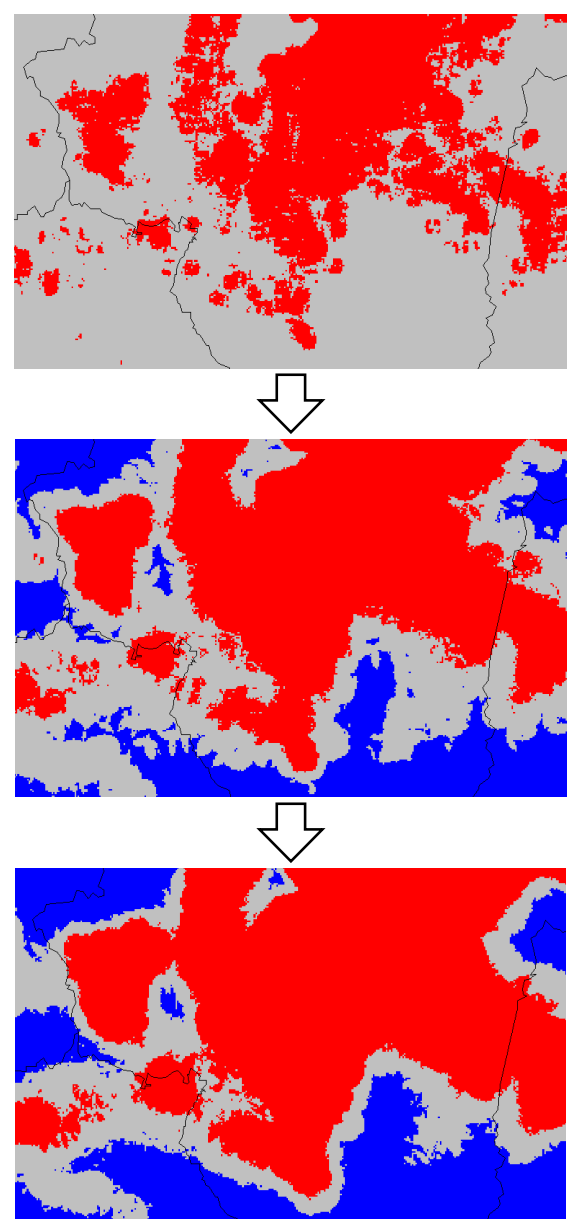

Figure 2. Fluctuation of spatial correlation/no spatial correlation areas depending on the distance parameter $d$ for local populations in Kagawa, 2015. Red and blue areas denote positive and negative correlation areas, respectively, while grey areas are no correlation areas.

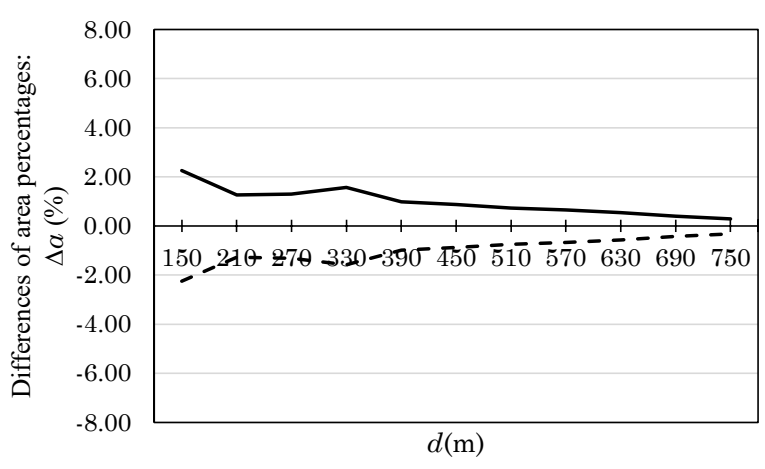

Figure 3. Fluctuation of the differences of spatial correlation/no spatial correlation areas between contiguous distances for local population in Kagawa, 2015. A solid line means $\Delta a$ of positive spatial autocorrelation areas, while a dotted line is that of negative and no spatial autocorrelation areas. 
the fluctuation of the differences of spatial correlation/no spatial correlation areas between contiguous distances for local population in Kagawa, 2015 as an example. The convergence of $\Delta a$ appears in Figure 3. We researched the fluctuations and convergences of $\Delta a$ and obtained the maximum of distances $d$ $\left(d_{\max }\right)$ for 4 kinds of local populations, respectively, in a case where $\Delta a$ shows less than $0.5 \%$. Table 1 indicates $d_{\max }$ derived from the research of the difference of the fluctuations.

\begin{tabular}{ccc}
\hline Area & Year & $d_{\max }(\mathrm{m})$ \\
\hline \multirow{2}{*}{ Osaka } & 1995 & 690 \\
& 2015 & 630 \\
\multirow{2}{*}{ Kagawa } & 1995 & 210 \\
& 2015 & 570 \\
\hline
\end{tabular}

Table 1. Maximum of distances $d\left(d_{\max }\right)$ derived from the convergence of $\Delta a$.

For detecting the phenomenon of "Urban spongification", it is required to analyse spatial features in a densely populated area because partially lower population density in an urbanized area potentially could be the sign of deterioration. We then focused attention on the occurrence of positive spatial autocorrelation areas along the distance parameter $d$. Figure 4 reveals the conceptual diagram with respect to the detection of the spatial features of local populations. Based on Figure 2, the area being within distance $d_{\max }$ of point $i$, showing positive autocorrelation, generally covers the positive areas being within distance $d_{\max -1}$ or less of $i$ because $\mathrm{G}$ statistics are basically derived from the summation of local populations within $d$ of $i$ (see Figures $4 \mathrm{a}$ and $4 \mathrm{~b}$ ). As the distance decreases from $d_{\max }$, a change of statistics (from positive spatial autocorrelation to no spatial autocorrelation) happens in distance $d_{n}$ at some points (see Figures $4 \mathrm{~b}$ and $4 \mathrm{c}$ ). We therefore define the distance $d_{n}$ as the Ambiguity of Spatial scale in a densely Populated area (ASP) since the null hypothesis that the set of local populations within $d_{n}$ of location $i$ is a random sample is not rejected even though positive spatial autocorrelations are consecutively shown in distance between $d_{\max }$ and $d_{n+1}$. In other words, there could be lower population density within distance $d_{n}$ in spite of the fact that there are significantly larger local populations within $d_{\max }$ of point $i$. The proposed ASP then makes possible to the detection of the sizes of population decline spots as well as their locations. These spatial features would indicate the sign of the phenomenon of "Urban spongification".

ASP is defined on the assumption that positive spatial autocorrelation is not shown in distance between $d_{n}$ and $d_{\text {min }}$. For proving the assumption, we calculated the occurrence of no/negative spatial autocorrelation in distance between $d_{n}$ and $d_{\text {min }}$. Table 2 reveals the relative frequency of occurrence of positive correlation within $d_{n}$ or less in Kagawa, 2015 as an instance. The interval of $d$ shows $60 \mathrm{~m}$ as the calculation was carried out by mesh-by-mesh basis. The relative frequency shows less than $1.3 \%$. We then ignored these cases because they seemed to be based on the uncertainty, i.e. georeferencing error of data, information losses of vector/raster conversion, and other issues of geographical data generations.
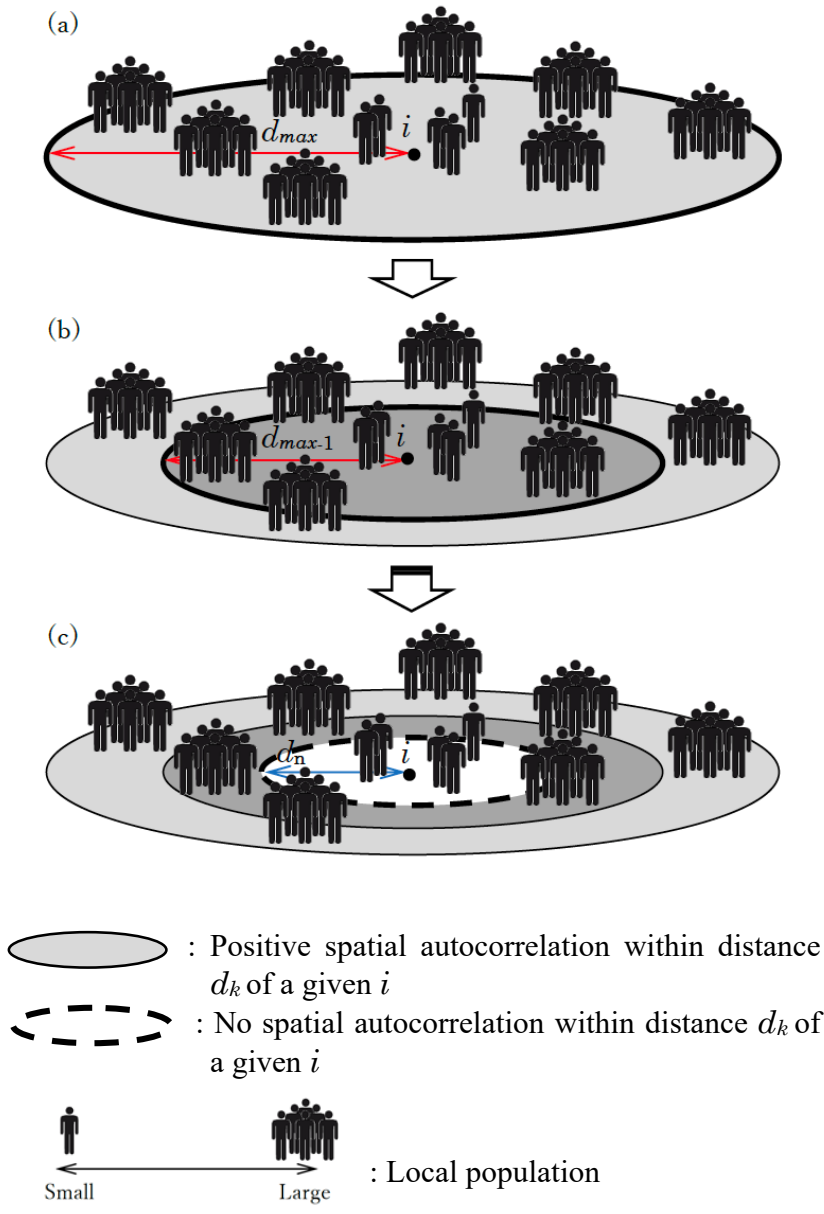

Figure 4. Conceptual diagram with respect to the detection of the spatial features of local populations.

\begin{tabular}{cc}
\hline$d(\mathrm{~m})$ & $\begin{array}{c}\text { Relative frequency of occurrence } \\
\text { of positive correlation } \\
\text { within } d_{n} \text { or less }\end{array}$ \\
\hline 90 & $1.19 \%$ \\
150 & $1.22 \%$ \\
210 & $0.90 \%$ \\
270 & $0.70 \%$ \\
330 & $0.54 \%$ \\
450 & $0.33 \%$ \\
510 & $0.00 \%$ \\
\hline
\end{tabular}

Table 2. Relative frequency of the occurrence of positive correlation within $d_{n}$ or less in Kagawa, 2015.

\section{RESULTS AND DISCUSSION}

\subsection{Results of Spatial Analysis}

Figure 5 shows the results of the spatial analysis we have developed. Coloured areas in Figure 5 mean densely populated areas because positive spatial autocorrelations are shown within distance $d_{\max }$ in the areas. Gradations in colour from yellow to red denote the fluctuation of $\mathrm{ASP} . \mathrm{ASP}_{0}$ means that larger local populations exist from the narrowest range to the widest range $\left(d_{\max }\right)$, while red hue of areas indicates large ASP. It is shown 


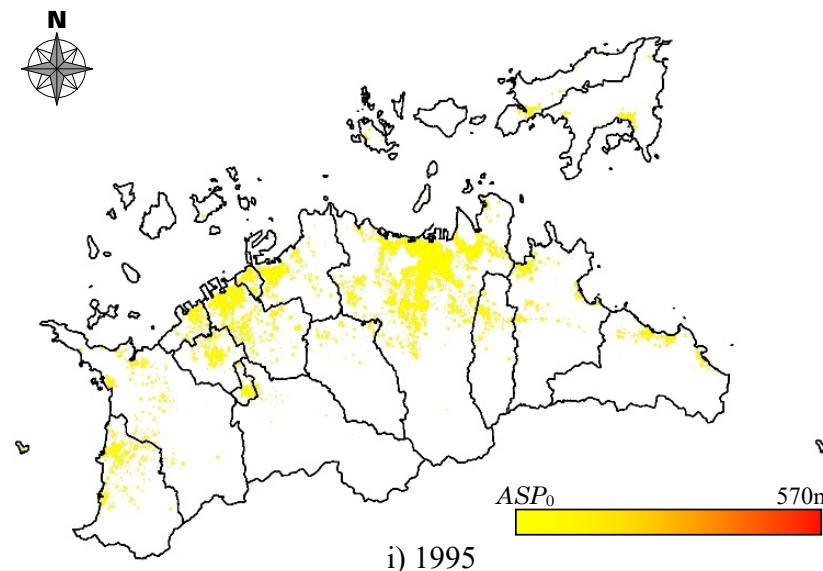

(a) Kagawa

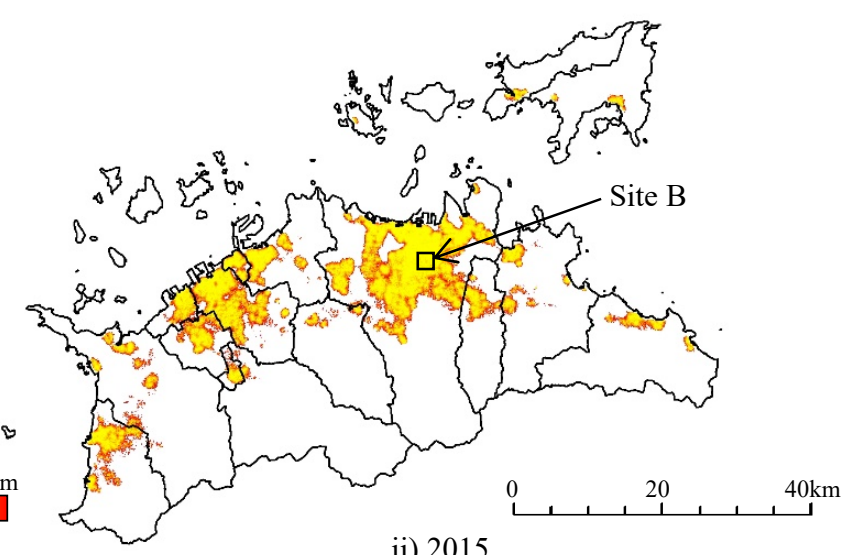

ii) 2015

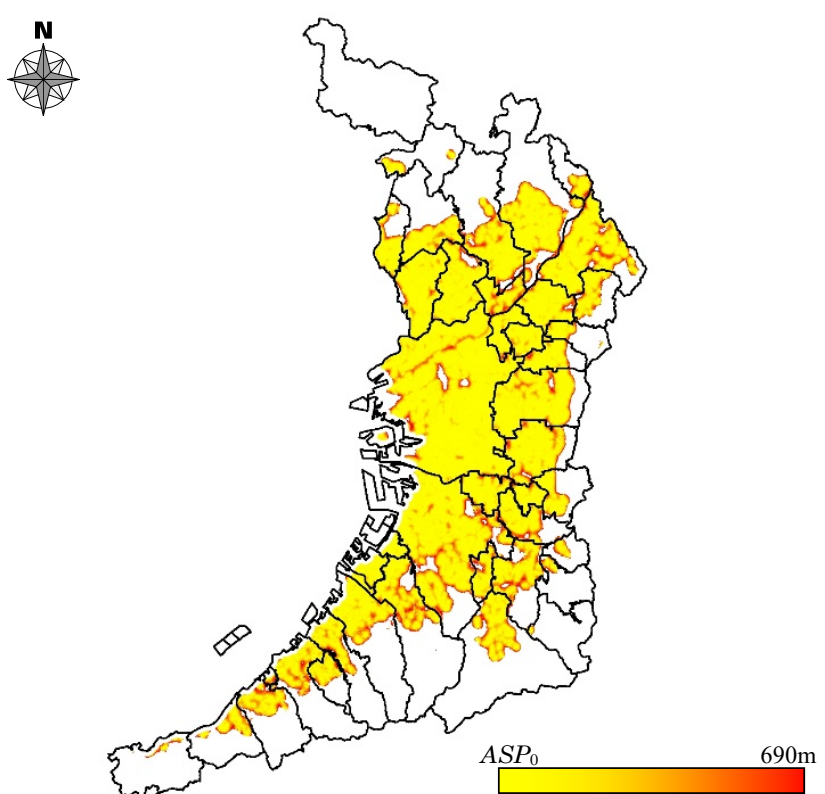

i) 1995

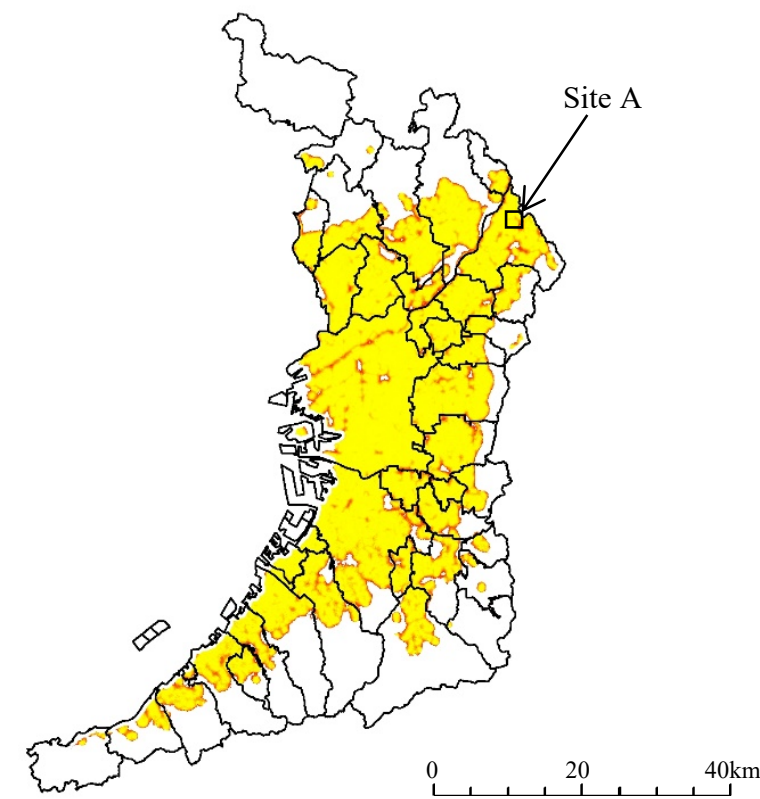

ii) 2015

(b) Osaka

Figure 5. Results of the spatial analysis we have developed: distributions of ASP.

that large ASPs are distributed principally around the edges of the coloured areas. There are also small red parts in the middle of the study areas: Osaka in 1995, 2015, and Kagawa 2015.

\subsection{Relationship between ASP and Urban Structure}

ASP obtained from spatial statistics shows the spatial feature value of local population distributions. Relationship between ASP and urban structure then should be clarified for the analysis of "Urban spongification". We examined the relationship from two viewpoints: local population density and land use.
3.2.1 Local Population Density: ASP suggests the size of area where larger local populations do not exist. Relationship between ASP and population density, however, is not still illuminated. We therefore defined population density within ASP as the Local Population density within ASP (LPA), and analysed the variation of LPA according to ASP. Figure 6 reveals the relative frequency distributions of LPA for individual ASPs in Osaka, 2015 as an example. In $\mathrm{ASP}_{0}$, the pattern of the histogram shows almost symmetric. According to the increase of ASP, the histograms gradually become skewed right. This tendency was also confirmed in other cases: Osaka 1995, Kagawa 1995 and 2015. It appears that ASP could contribute to the detection of lower population density districts within a densely populated area. The larger ASP also seems to indicate the locations of much lower population density districts. It is thus obvious that proposed ASP would be able to provide the analysis of "Urban spongification" with significant information regarding the spatial scale as well as location of lower populated districts. 


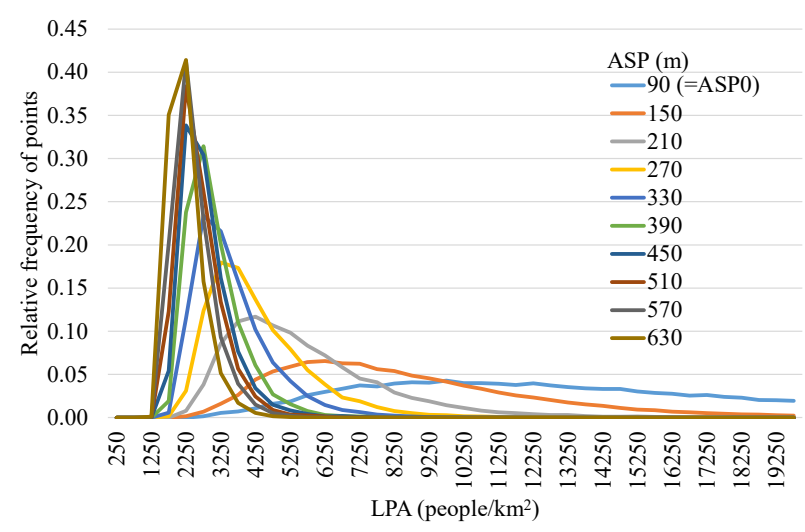

Figure 6. Relative frequency distributions of LPA for individual ASPs in Osaka 2015.

3.2.2 Comparison of ASP and Land Use: Figure 7 reveals zoomed-in images of ASP of Figure 5 and aerial photographs for the comparison of the results of the spatial analysis and current land use. We can recognise the large ASP $(=300 \mathrm{~m})$ is obtained where there are large agricultural fields and a few buildings and houses in the dotted circle with radius ASP in Figure 7a. There are also wide industrial areas with larger ASPs in the lower right of Figure 7a. ASP seems to depend on the current status of land use around a centre pixel. On the other hand, in Figure 7b, it is difficult to interpret the feature of land use around the dotted circle with radius ASP though there are buildings, houses, and small patches of agricultural fields and parking lots in the whole area of Figure $7 \mathrm{~b}$. Around the
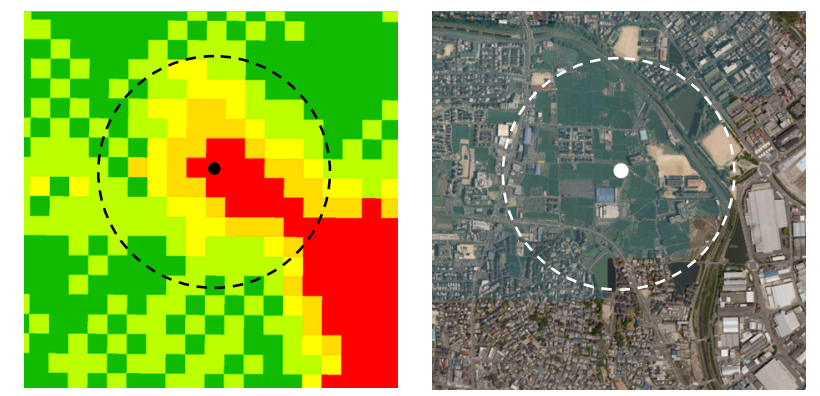

(a) Site A in Osaka
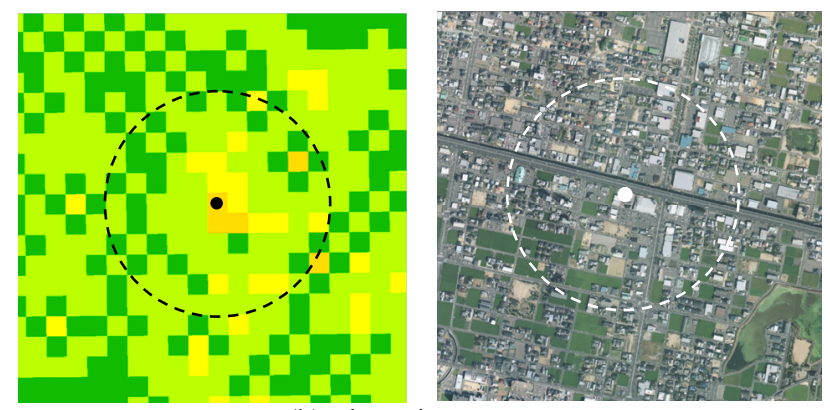

(b) Site B in Kagawa

: ASP $_{0} \square: 150 \mathrm{~m}-210 \mathrm{~m} \quad: 270 \mathrm{~m} \quad \square: 330 \mathrm{~m} \quad \square: 390 \mathrm{~m}-$

Figure 7. Comparison between the large ASPs and aerial photographs in enlarged views of study areas. Dotted lines show the circles with radius ASP (= $330 \mathrm{~m}$ ) which is indicated with colour of centre pixels in ASP images. centre pixel, various ASPs are generated in this site. ASP also seems to include the spatial feature of local population distributions, which does not appear on a land use status.

\subsection{Discussion}

ASP and LPA have the potential to contribute to the detection of lower population density spots in urbanized areas. Based on the tendency in Figure 6, it would be appropriate to apply ASP and LPS simultaneously to the spatial analysis of local population distributions. For the application to the monitoring of local population distributions, the detection of the fluctuations of the spatial feature needs to be discussed.

3.3.1 Spatial Variation between 2 Periods: As the first step of the discussion, we analysed the difference of both ASP and LPA between 2 periods: 1995 and 2015. Figure 8 shows the concept of spatial variation based on the difference between 2 periods. The variation of LPA is shown in the horizontal axis, while that of ASP is displayed in the vertical axis. Figure 8 also indicates the conceptual drawings of spatial variation patterns in some quadrants. The spatial variation pattern in the lower left quadrant shows the extension of ASP: the area of lower population density is enlarged. This therefore means the sign of the expansion of "Urban spongification". In the upper right quadrant, the spatial pattern is symmetric with respect to the point of intersection of the 2 axes: ASP is decreasing as well as LPA is increasing. It is theoretically impossible to describe the spatial patterns in the quadrants of upper left and lower right at this stage. We used these 4 quadrants to examine the applicability of this simple difference approach to the spatial features.

3.3.2 Application of the Difference Approach: We applied the difference approach to ASP and LPA between 1995 and 2015, in Osaka and Kagawa, respectively. Figure 9 reveals the bubble charts consisting of the number of points and the variations of LPA and ASP. The size of bubble shows the number of points fell into each interval: ASP of $60 \mathrm{~m}$ and LPA of 2500 people $/ \mathrm{km}^{2}$. In Figure 9a, there are a lot of frequency in the upper left quadrant. A leading cause of this result seems to be the difference of $d_{\max }$ between 1995 and 2015 in Kagawa. The spatial pattern of ASP image in 1995 differs considerably from that of ASP image in 2015 (see Figure 5a). It does not seem to be appropriate to apply the simple difference approach in a case where the fluctuation range of spatial pattern is large. Advanced difference approach including the standardization of ASP related to LPA would expect to be developed. In Figure $9 \mathrm{~b}$, larger frequency data are almost distributed in the quadrants of upper right and lower left and on the horizontal axis because the rate of change of total population is less than $0.5 \%$ in Osaka. 


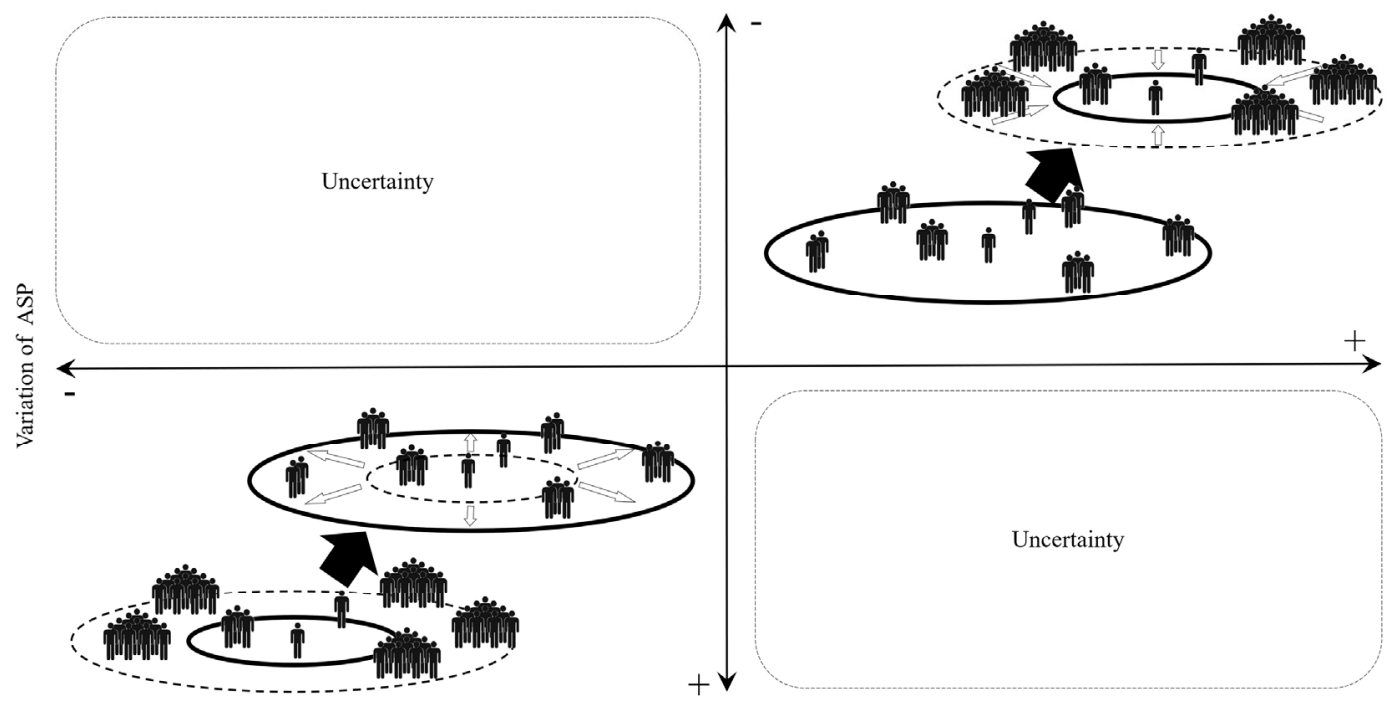

Variation of LPA

Figure 8. Concept of spatial variation based on the difference among 2 periods. Solid circles denote areas within ASP.

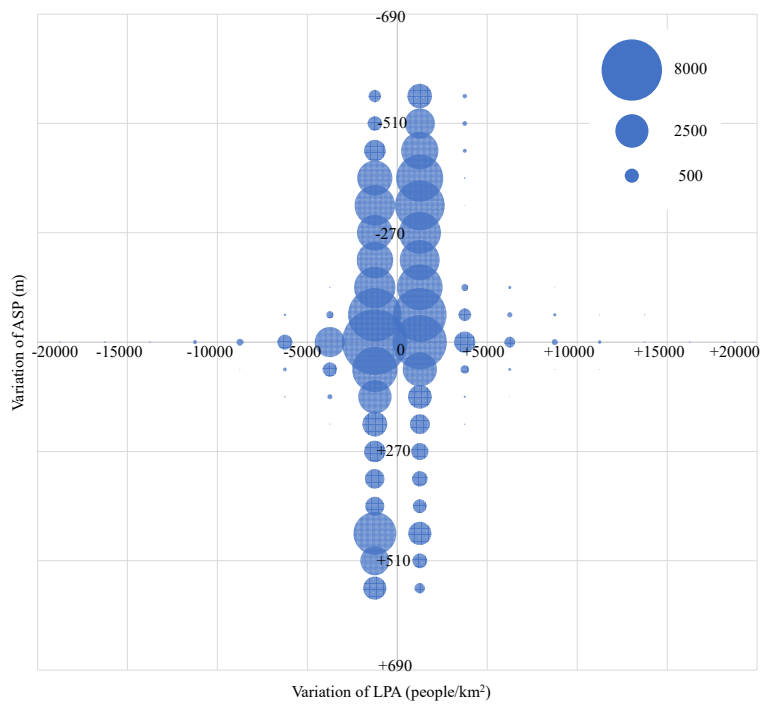

(a) Kagawa

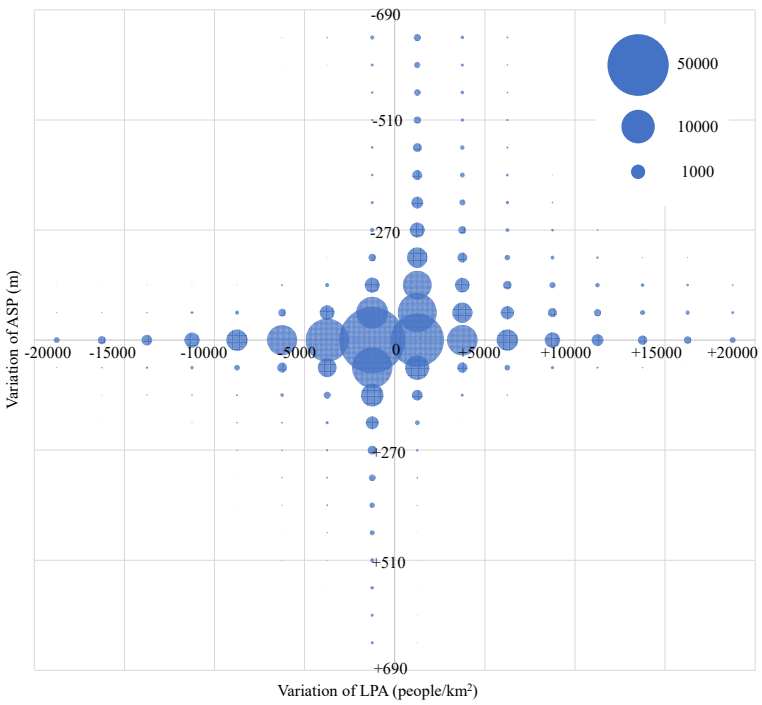

(b) Osaka

Figure 9. Bubble charts consisting of the number of points and the variations of LPA and ASP. The size of bubble shows the number of points fell into each interval: ASP of $60 \mathrm{~m}$ and LPA of 2500 people $/ \mathrm{km}^{2}$.

3.3.3 Visualization of Spatial Variation: We mapped the spatial variations between 1995 and 2015 in Osaka based on the division of Figures 8 and 9b. Figure 10 indicates the spatial variation map of local population distributions between 1995 and 2015 in Osaka. The status of local spatial variations is described in colours gradated by the transformation of the 2 variations into hue and saturation, as in the legend of Figure 10. In Figure 10, isabella areas (LPA variation of -200 to -7500 people $/ \mathrm{km}^{2}$ and ASP variation of $0 \mathrm{~m}$ ) dominates, while blue hot spots (LPA variation of more than +15000 people $/ \mathrm{km}^{2}$ and ASP variation of $0 \mathrm{~m}$ ) are scattered around the centre of the study area. We can see several light green areas (LPA variation of less than -7500 people $/ \mathrm{km}^{2}$ and ASP variation of more than $+240 \mathrm{~m}$ ) generated in the centre and edge of the coloured area.
3.3.4 Comparison of the Spatial Variations and the Fluctuations of Local Population Distributions: Figures 11, 12,13 , and 14 show the partial images of sites C, D, E, and F of Figure 10, respectively, along with aerial photographs and local population distributions. Dashed lines denote the circles with radius ASP in 1995, while dotted lines means the circles with radius ASP in 2015. In the dark purple area in site $C$ on the spatial variation map, it is confirmed that there are high-rise apartments around the centre point in Figure 11b. At the blue hot spots in Figure 10, we could also see high-rise condominium is often located. Likewise, we could see the areas coloured yellow in Figure 10 were almost in course of redevelopment. Partial increase/decrease of local populations related to a fixed-area development/redevelopment seems to be classified around the horizontal axis of Figure 8 and the legend of Figure 10. About a purple area in site D on the spatial variation map, corresponding to upper right quadrant of Figure 


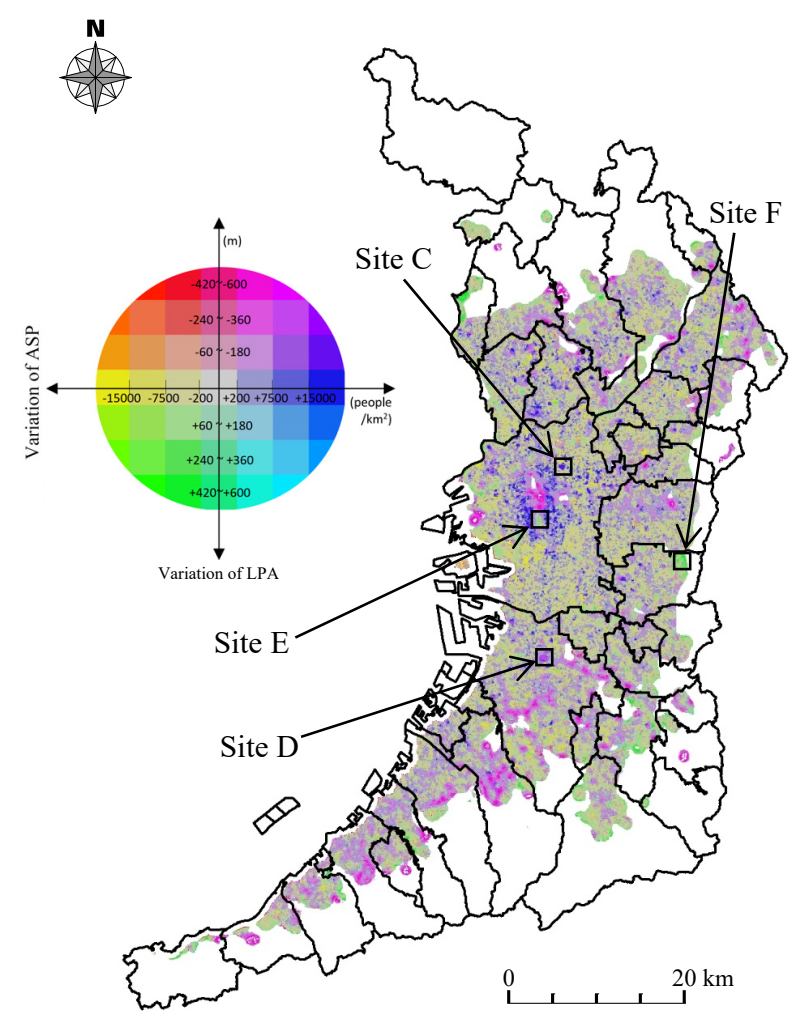

Figure 10. Spatial variation map of local populations among 1995 and 2015 in Osaka.

8, a lot of local populations increasing from 1995 to 2015 distribute around the centre point in Figures $12 \mathrm{c}$ and $12 \mathrm{~d}$. There have been a district planning under a land readjustment project for recent 30 years in this area. ASP is decreasing since the areal improvement causes the attraction of larger local populations. Site E of Figure 10 is located in centre of the Osaka city. There are so many buildings for business and commercial use in this area (see Figure 13b). The daytime population is then very large usually. A hollowing out of nighttime population therefore seemed to occur during 20 years because the local population data used in this study is generated from nighttime population. Figure 14 shows the result and aerial photograph of a suburban area. The density of local population distributions is low depending on land use status: there are small residential districts around agricultural fields. On the basis of the comparison of local populations between 1995 and 2015 in Figures 14c and 14d, typical population decline seems to occur in this area. These phenomena in Figures 13 and 14 differ from "Urban spongification". It is, however, obvious that the spatial variation consisting of ASP and LPA variations seems to have an applicability to the detection of the sign of local population dynamics, such as "Urban spongification". In cases where ASP is increasing in the Residence Attraction Districts, the necessity of the revision of the measure may be suggested against the deterioration of the districts under the population decline.

\section{CONCLUSIONS}

In this study, we applied spatial autocorrelation method to local populations for the detection of the sign of population dynamics, especially "Urban spongification", which potentially reinforces the run-down of a district. Population decline in urbanized areas usually generates vacant land and vacant house a lot. The monetary value of areas which includes much vacant land and vacant houses is decreasing and this again reinforces the spiral of decline. Based on the relationship between distance parameter and positive autocorrelation areas, we defined the spatial-scale feature of local population distributions as Ambiguity of Spatial scale in a densely Populated area (ASP). We also defined population density within ASP as Local Population density in the range of ASP (LPA). Through the analysis of the relationship between ASP and LPA, ASP could contribute to the detection of lower population density districts within a densely populated area. For the application to the monitoring of local population distributions, we examined the simple difference approach using ASP and LPA in 1995 and 2015 as a first step. As the result, it was apparent that the spatial variations derived from the variations of ASP and LPA could suggest the spatial features of local population dynamics. It is desirable to be able to detect spatial variations over multiple spans of time since the process of population dynamics is useful for the evaluation of the current measures. Proposed method has a possibility to be applied to the detection.

As problems remaining for future research, it should be apparent how to analyse spatial features of local population dynamics in a case where the fluctuation range of spatial pattern is large. In general, population decline in a provincial area occurs sharply. More research is needed in the analysis of spatial feature fluctuations. It is also required to clarify what ASP represents with respect to urban structure. ASP derived from statistical test for local populations is not necessarily related to the factors of urban structure. Procedures for the urban structure analysis, which are composed of spatial analysis of land use, urban facilities and development/redevelopment project as well as local population dynamics, should be discussed.

\section{ACKNOWLEDGEMENTS}

This work was supported by JSPS KAKENHI Grant Number JP20K04727. The basic map of study area and aerial photographs are adopted from GSI tiles (https://maps.gsi.go.jp/development/ichiran.html) provided by the Geospatial Information Authority of Japan. We would like to thank the helpful comments from anonymous reviewers.

\section{REFERENCES}

Getis, A., Ord, J. K., 1992: The analysis of spatial association by use of distance Statistics. Geographical Analysis, 24(3), 189-206.

Kumagai, K., 2011: Verification of the analysis method for extracting the spatial continuity of the vegetation distribution on a regional scale. Computers, Environment and Urban Systems, 35, 399-407.

Kumagai, K., Uematsu, H. Matsuda, Y., 2017: Advanced spatial analysis for vegetation distributions aimed at introducing smarter city shrinkage. Planning Support Science for Smarter Urban Futures, Lecture Notes in Geoinformation and Cartography, Springer International Publishing, 469-489.

Kutsuzawa, R., 2016: The effect of compact city on cities budget - the analysis by standard distance. Urban Housing Sciences, 2016(95), 142-150 (in Japanese). 


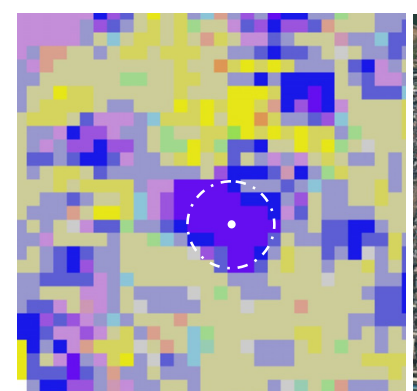

(a) Spatial variation

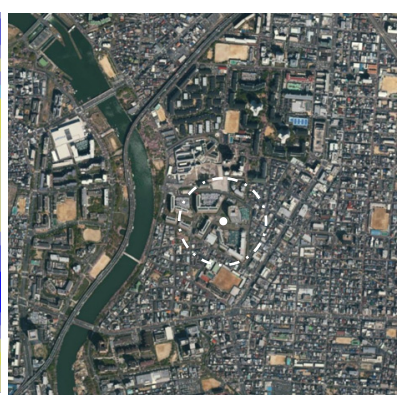

(b) Aerial photo

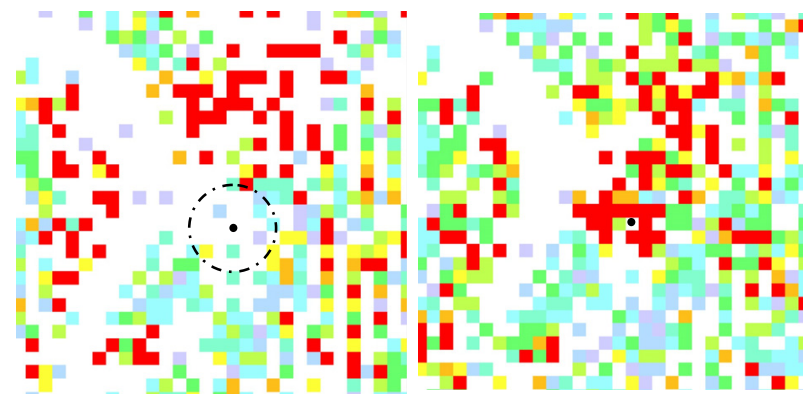

$\begin{array}{ll}\text { (c) Local populations in1995 } & \text { (d) Local populations in } 2015\end{array}$ Local population: ${ }^{0}$ $200-$
Figure 11. Partial image of site $C$ in Figure 10 along with aerial photograph and local population distributions. Dashed circle denotes ASP $(=210 \mathrm{~m})$ in 1995. In 2015, circle does not appear ( $\left.\mathrm{ASP}_{0}\right)$.

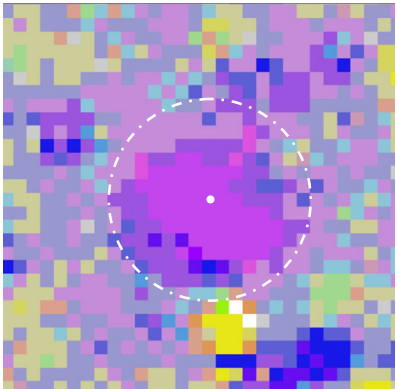

(a) Spatial variation

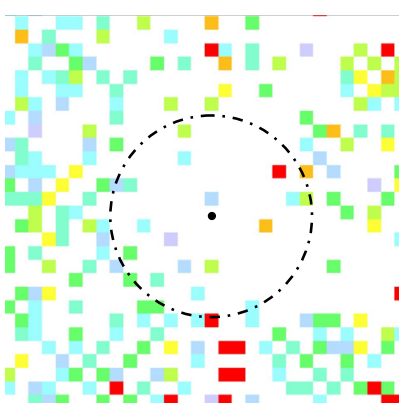

(c) Local populations in 1995

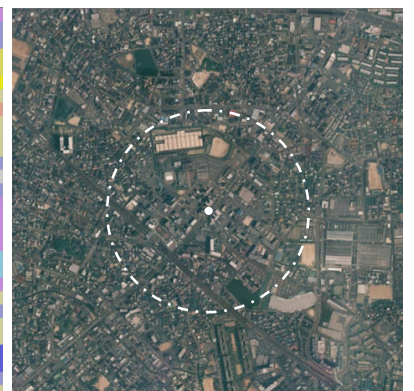

(b) Aerial photo

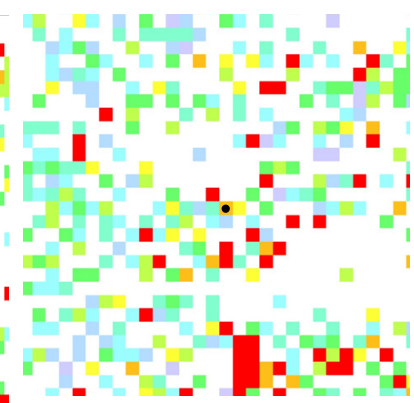

(d) Local populations in 2015

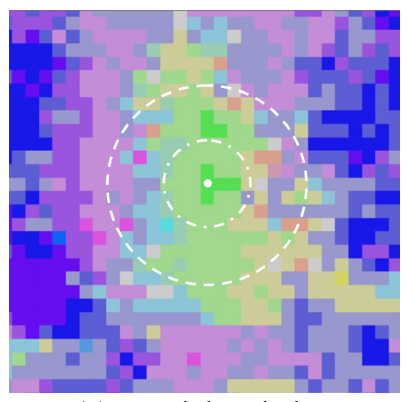

(a) Spatial variation

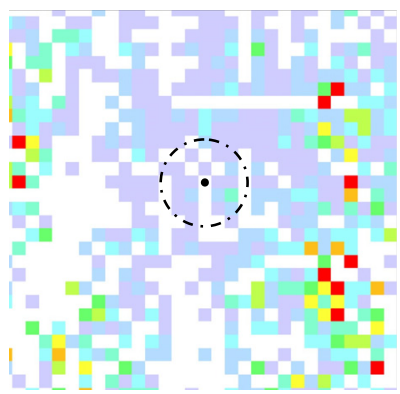

(c) Local populations in 1995

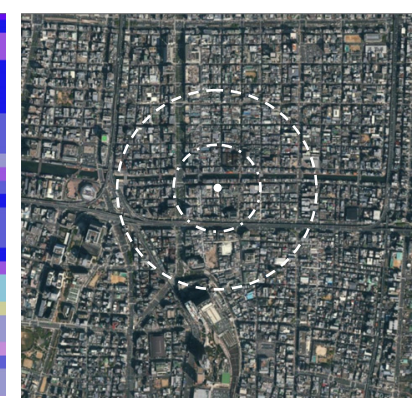

(b) Aerial photo

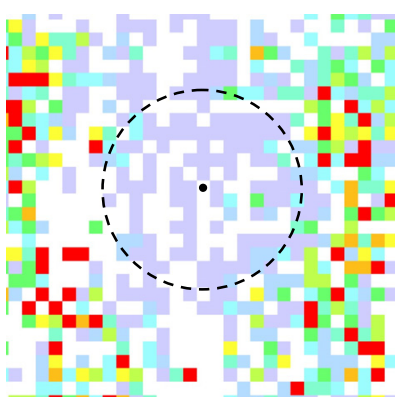

(d) Local populations in 2015

200 -

Figure 13. Partial image of site $\mathrm{E}$ in Figure 10 along with aerial photograph and local population distributions. Dashed circle denotes ASP $=210 \mathrm{~m}$ in 1995, while dotted circle means ASP $=450 \mathrm{~m}$ in 2015.

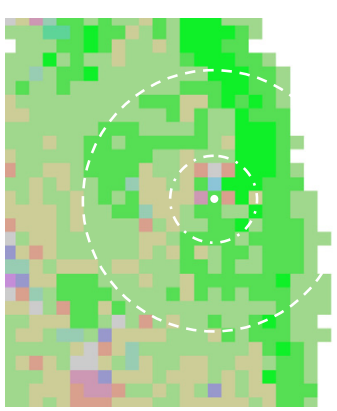

(a) Spatial variation

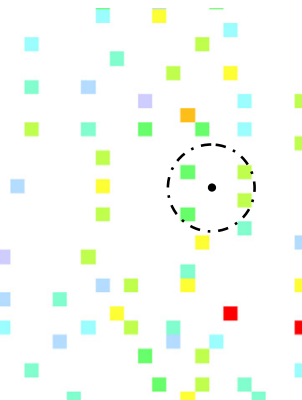

(c) Local populations in 1995

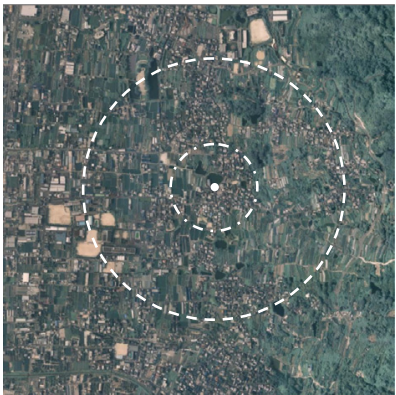

(b) Aerial photo

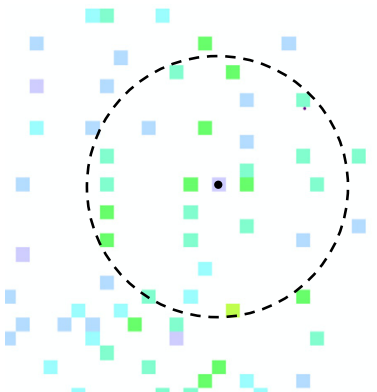

(d) Local populations in 2015

Local population: ${ }^{0}$ 200 -

Figure 14. Partial image of site $\mathrm{F}$ in Figure 10 along with aerial photograph and local population distributions. Dashed circle denotes ASP $=210 \mathrm{~m}$ in 1995 , while dotted circle means ASP $=570 \mathrm{~m}$ in 2015. 
Ministry of Land, Infrastructure, Transport and Tourism, 2014: Promotion of Urban Renovation and Compact Cities. http://www.mlit.go.jp/common/000996976.pdf $\quad(1 \quad$ February 2021).

Ministry of Land, Infrastructure, Transport and Tourism, 2019: White Paper on Land, Infrastructure, Transport and Tourism in Japan, 2019. https://www.mlit.go.jp/en/statistics/white-papermlit-2019.html (1 February 2021).

National Institute of Population and Social Security Research, 2012: Population Projections for Japan (January 2012). http://www.ipss.go.jp/sitead/index_english/esuikei/gh2401e.asp (1 February 2021).

Ord, J. K. Getis, A., 1995: Local Spatial Autocorrelation Statistics: Distributional Issues and an Application. Geographical Analysis, 27(4), 286-306. 